\title{
Percepção dos responsáveis de crianças e adolescentes sobre prescrição da cadeira de rodas e satisfação com o equipamento
}

\section{Child and adolescent primary caregiver's perception of wheelchair prescription and equipment satisfaction}

\author{
Alessandra Cavalcanti' ${ }^{1}$, Eduarda Caroline Marques Borges ${ }^{2}$, Bruna Correa Magalhães ${ }^{2}$, \\ Edinara Kososki ${ }^{2}$, Alberto Luiz Aramaki ${ }^{1}$, Simone Gonçalves Silva e Silva ${ }^{3}$
}

http://dx.doi.org/10.11606/issn.2238-6149.v29i1p27-33

Cavalcanti A, Borges ECM, Magalhães BC, Kososki E, Aramaki AL, Silva-Silva SG. Percepção dos responsáveis de crianças e adolescentes sobre prescrição da cadeira de rodas e satisfação com o equipamento. Rev Ter Ocup Univ São Paulo. 2018 jan.-abr.;29(1):27-33.

RESUMO: Objetivo: Investigar a percepção dos pais/responsáveis sobre o motivo da solicitação de cadeira de rodas para suas crianças/ adolescentes e descrever se existe conforto ou desconforto deles na cadeira, assim como os itens de satisfação ou não no equipamento concedido em um serviço de concessão no município de Uberaba, MG. Métodos: Participaram do estudo 29 crianças/adolescentes com faixa etária mínima de três anos e máxima de 17. Para a coleta dos dados foram aplicados um instrumento estruturado e um roteiro de entrevista. Resultados: A idade média dos participantes foi de 10,03 anos, com predomínio de crianças. O diagnóstico clínico que prevaleceu foi paralisia cerebral. Os resultados mostraram que o motivo da solicitação de prescrição da cadeira de rodas foi mobilidade (46,7\%). 86,2\% dos pais/responsáveis apontaram perceber desconforto da criança/ adolescente durante o uso da cadeira de rodas. Uma maioria declarou estar satisfeito com à mobilidade que a cadeira fornece, e se dizem insatisfeitos com os acessórios (cinto, sistema de sentar, opção de reclínio, dentre outros) da cadeira de rodas. Conclusões: Frente aos apontamentos de desconforto e insatisfação conclui-se que é importante rever o processo de prescrição da cadeira de rodas no serviço de concessão estudado e o acompanhamento das crianças/adolescentes de forma mais próxima, melhorando o serviço de prescrição e seleção dos equipamentos.

DESCRITORES: Equipamentos de autoajuda; Cadeiras de rodas; Centros de reabilitação; Criança; Adolescente.
Cavalcanti A, Borges ECM, Magalhães BC, Kososki E, Aramaki AL, Silva-Silva SG. Child and adolescent primary caregiver's perception of wheelchair prescription and equipment satisfaction. Rev Ter Ocup Univ São Paulo. 2018 Jan.-Apr.;29(1):27-33.

\begin{abstract}
Objective: To investigate the perception of the parents/ guardians about the reason for requesting a wheelchair for their child and adolescent and to describe if there is comfort or discomfort in the wheelchair, as well as the items of satisfaction or dissatisfaction in the equipment granted in a concession service in the city of Uberaba, MG. Methods: Twenty-nine child and adolescent with a minimum age of three years and a maximum of 17 participated in the study. For the data collection, a structured instrument and an interview script were applied. Results: The mean age of participants was 10.03 years, with a predominance of children. The clinical diagnosis that prevailed was cerebral palsy. The results showed that the reason for requesting a wheelchair prescription was mobility (46.7\%). 86.2\% of the parents/guardians indicated that they perceive discomfort of the child/adolescent during the use of the wheelchair. A majority stated that they were satisfied with the mobility the chair provides, but they also say they are dissatisfied with the wheelchair's accessories (seat belt, seating system, reclining option, among others). Conclusions: In the face of discomfort and dissatisfaction, it is concluded that it is important to review the wheelchair prescribing process in the concession service studied and the monitoring of the child/ adolescents more closely, improving the prescription and equipment selection service.
\end{abstract}

KEYWORDS: Self-help devices; Wheelchairs; Rehabilitation centers; Child; Adolescent.

Estudo desenvolvido pelo Laboratório Integrado de Tecnologia Assistiva da Universidade Federal do Triângulo Mineiro (LITA/UFTM), Uberaba (MG), Brasil - aprovado pelo Comitê de Ética em Pesquisa com Seres Humanos da Universidade Federal do Triângulo Mineiro de acordo com o parecer $n^{\circ} 1.560 .653$ de 2016 .

1. Departamento de Terapia Ocupacional da Universidade Federal do Triângulo Mineiro - UFTM. Pesquisadores do Laboratório Integrado de Tecnologia Assistiva (LITA/UFTM). Uberaba (MG), Brasil. ORCID: A. Cavalcanti - https://orcid.org/0000-0002-2306-2031; A. L. Aramaki - https://orcid.org/0000-0002-1740-6686. E-mail: alessandra.cavalcanti@uftm.edu.br; alberto_koston@hotmail.com

2. Terapeuta ocupacional. Pesquisadoras do Laboratório Integrado de Tecnologia Assistiva (LITA/UFTM). Uberaba (MG), Brasil. ORCID: E.C.M. Borges - https://orcid.org/0000-0002-4283-608X; B. C. Magalhães - https://orcid.org/0000-0002-3726-1680; E. Kososki - https:// orcid.org/0000-0003-0093-9547.E-mail: eduardacmarques.uftm@hotmail.com; brunacorrea.to@gmail.com; edinara_kososki@hotmail.com

3. Terapeuta ocupacional. Centro de Reabilitação do Complexo Hospitalar da Universidade Federal do Triângulo Mineiro. Uberaba (MG), Brasil. ORCID: https://orcid.org/0000-0002-3837-9250.E-mail: enomisilva@hotmail.com

Autor para correspondência: Alessandra Cavalcanti. Universidade Federal do Triângulo Mineiro, Unidade Centro Educacional. Departamento de Terapia Ocupacional. Av. Getúlio Guaritá, n 159, sala 329. Bairro Abadia, Uberaba, MG. CEP: 38025-440.Email: alessandra.cavalcanti@uftm.edu.br 


\section{INTRODUÇÃO}

cadeira de rodas é um dispositivo
de tecnologia assistiva com funções
diversas que visa fornecer adequação postural, possibilitar mobilidade funcional e assistir no transporte de pessoas com deficiência física. Quando adequadamente prescrita às necessidades da pessoa (criança, adolescente, adulto ou idoso), promove conforto, simetria e estabilidade na postura sentada, assim como favorece a autonomia e a independência do usuário na realização de suas atividades do dia a dia ${ }^{1}$.

Cooper ${ }^{2}$ sintetizou a importância do equipamento afirmando-o como essencial para a mobilidade na comunidade, para o envolvimento em atividades $\mathrm{e}$ tarefas necessárias e/ou desejadas e determinante na participação social. É o dispositivo que permite que pessoas com limitação na mobilidade sejam independentes para deslocamento, favorecendo oportunidade para desempenho de seus papéis na sociedade ${ }^{3}$.

Recentemente, os diferentes setores que compõem o cenário de fabricação, prescrição e uso da cadeira de rodas se voltaram para estudos e observações que envolvem os problemas apresentados nos diferentes modelos de cadeira de rodas, a frequência de reparo, os relatos de queda do dispositivo e as lesões por esforço repetitivo no membro superior $^{3,4,5}$.

Quando o usuário da cadeira de rodas é uma criança ou adolescente, os pais ou os tutores são os responsáveis pela demanda para aquisição do equipamento. A compra da cadeira de rodas pode ocorrer diretamente em lojas especializadas ou ser solicitada em um setor de concessão disponível pelo serviço público do município onde reside o usuário ${ }^{6}$. Em ambas situações são os pais ou os tutores que vão apresentar as necessidades sobre o equipamento para que ele seja então selecionado por um profissional habilitado. Ao longo da avaliação para prescrição e seleção de uma cadeira de rodas, a equipe deve reconhecer que a experiência de cada usuário é individual e única; portanto, a escuta sobre suas preferências, sobre suas necessidades e sobre o que é mais útil também guia o processo de escolha do equipamento mais adequado para cada caso ${ }^{7}$.

Deste modo, a participação dos usuários no processo de escolha, aquisição e verificação da qualidade de dispositivos de assistência é fundamental para a melhoria dos serviços que dispensam tecnologia assistiva. Neste contexto, o objetivo deste trabalho foi investigar a percepção dos pais/ responsáveis sobre o motivo da solicitação de cadeira de rodas para suas crianças/adolescentes e descrever se existe conforto ou desconforto deles na cadeira, assim como quais são os itens de satisfação ou não no equipamento concedido em um serviço de concessão no município de Uberaba, MG

\section{PROCEDIMENTOS METODOLÓGICOS}

Estudo observacional transversal de natureza descritiva aprovado pelo Comitê de Ética em Pesquisa com Seres Humanos da Universidade Federal do Triângulo Mineiro por meio do parecer CAEE $\mathrm{n}^{\circ}$ 52673216.5.0000.5154 de 2016.

A amostra da pesquisa foi determinada a partir de estudo anterior ${ }^{8}$ (dados ainda não publicados), que possibilitou identificar a concessão de 76 cadeiras de rodas manuais modelo infantil para crianças/adolescentes no ano de 2014, por meio do programa do governo federal de "Concessão de órteses, próteses e meios auxiliares de locomoção ambulatoriais", fornecida pelo Sistema Único de Saúde (SUS) em um serviço de reabilitação em Uberaba, MG.

$\mathrm{Na}$ cidade de Uberaba, MG, este serviço localizase na unidade de reabilitação ambulatorial do Hospital de Clínicas da Universidade Federal do Triângulo Mineiro por meio de parceria entre a Instituição de Ensino e a Secretaria de Saúde do Município. Semanalmente, são realizadas avaliações e prescrições de equipamentos de tecnologia assistiva (cadeiras de rodas, adaptação postural em cadeira de rodas, órteses, próteses e materiais especiais (OPM)) para serem dispensados anualmente por meio do SUS para 19 municípios que compõem a macrorregião do Triângulo Sul do Estado de Minas Gerais, incluindo a cidade de Uberaba.

Neste estudo foram excluídos 47 usuários de cadeiras de rodas: 39 usuários que não residem no município de Uberaba, sete usuários que não foram localizados no endereço e telefone informado no prontuário e um usuário que foi a óbito. Portanto, a amostra final do estudo foi composta por 29 usuários de cadeira de rodas. Os informantes foram o pai, a mãe ou o responsável pela criança/adolescente usuária da cadeira de rodas. Nenhum participante contatado recusou participar do estudo e todos consentiram, assinando o Termo de Consentimento Livre e Esclarecido (TCLE) no momento inicial da coleta de dados.

A coleta de dados foi realizada no domicílio dos participantes, e uma equipe constituída de cinco entrevistadores membros do Laboratório Integrado de Tecnologia Assistiva da Universidade Federal do Triângulo Mineiro foram habilitados para a aplicação dos instrumentos e foram instruídos e treinados quanto à maneira de abordar os participantes e quanto à eticidade da pesquisa. A coleta de dados ocorreu entre os meses de abril a agosto de 2016. 
Para a coleta de dados foram utilizados dois instrumentos. O primeiro referente ao nível econômico segundo o Critério de Classificação Econômica Brasil CCEB da Associação Brasileira de Empresas de Pesquisa$\mathrm{ABEP}^{9}$ que explicita a renda média familiar do participante por classes: A ( R \$ 20.272,56), B1 ( $\mathrm{R} \$ 8.695,88), \mathrm{B} 2(\mathrm{R} \$$ 4.427,36), C1 (R\$2.409,01), C2 (R\$ 1.446,24), D-E (R\$ $639,78)$. E, outro instrumento: um roteiro de entrevista adaptado de McDonald, Surtees e Wirz 6 , constituído por uma parte genérica que caracteriza o participante, outra parte com questões fechadas para registro sobre o motivo da solicitação da cadeira de rodas (gerenciamento da postura, mobilidade, facilidade para uso e transporte) para a criança/adolescente, histórico do tempo de uso da cadeira de rodas em um dia típico (menos que uma hora, de uma a três horas, de três a seis horas, de seis a dez horas, mais que dez horas) e tempo em que a mesma foi concedida (menos que seis meses, de seis a doze meses, de doze a dezoito meses, de dezoito meses a dois anos, mais que dois anos). A segunda parte deste instrumento é composta por perguntas abertas, para apontamento em no máximo três itens sobre quando percebem conforto e desconforto de suas crianças/adolescentes na cadeira, e quais itens geram satisfação ou insatisfação no equipamento concedido.

Na parte genérica do questionário, o gerenciamento da postura revela a função da cadeira de rodas para auxiliar no posicionamento sentado. A mobilidade se refere a locomoção do usuário para se envolver em atividades e ter participação, permitindo seu deslocamento dentro e fora de casa para seu envolvimento em ocupações e tarefas. A facilidade para uso aponta para a particularidade de manejo e uso da cadeira de rodas, e, o transporte expressa a condução esporádica do usuário de um lugar para outro.

As variáveis qualitativas (nível econômico, gênero, diagnóstico clínico e motivo da prescrição) provenientes das questões fechadas foram submetidas à análise descritiva por meio de frequência absoluta e relativa, e das questões discursivas (satisfação, conforto, insatisfação e desconforto) foram categorizadas pela técnica de análise de conteúdo descrita por Bardin ${ }^{10}$ e apresentadas em termos de frequência de aparição. A variável quantitativa idade foi analisada por média e desvio padrão, e as variáveis de tempo de uso da cadeira de rodas em um dia e tempo de concessão foram analisadas descritivamente por meio de frequência absoluta e relativa.

\section{RESULTADOS}

As características (faixa etária, gênero, diagnóstico clínico e renda média domiciliar) das crianças e adolescentes usuárias das cadeiras de rodas manual modelo infantil que compuseram a amostra podem ser observadas na Tabela 1. A idade média foi de 10,03 anos $(\mathrm{DP}=4,15)$, sendo a maior idade 17 anos e a menor três anos. Quanto ao gênero, 51,7\% são meninas e 48,3\% são meninos. Em relação ao diagnóstico clínico, predominam os casos de paralisia cerebral $(82,8 \%)$. E em relação à renda média dos participantes, verificouse que $37,9 \%$ pertencem a classe $\mathrm{C} 1$, portanto possuem como estimativa de renda domiciliar mensal o valor de $\mathrm{R} \$ 2.409,01$.

Tabela 1 - Características dos usuários de cadeira de rodas manual infantil $(n=29)$

\begin{tabular}{|c|c|c|}
\hline Variável & n & $\%$ \\
\hline \multicolumn{3}{|l|}{ Faixa Etária } \\
\hline $02-06$ & 08 & 27,6 \\
\hline $07-11$ & 08 & 27,6 \\
\hline $12-18$ & 13 & 44,8 \\
\hline \multicolumn{3}{|l|}{ Gênero } \\
\hline Feminino & 15 & 51,7 \\
\hline Masculino & 14 & 48,3 \\
\hline \multicolumn{3}{|l|}{ Diagnóstico Clínico } \\
\hline \multicolumn{3}{|l|}{ Paralisia cerebral } \\
\hline Tetraparesia & 19 & 65,5 \\
\hline Diparesia & 04 & 13,8 \\
\hline Atáxica & 01 & 03,4 \\
\hline Mielomeningocele & 03 & 10,3 \\
\hline Malformação Congênita & 01 & 03,4 \\
\hline Câncer & 01 & 03,4 \\
\hline \multicolumn{3}{|l|}{ Nível Econômico } \\
\hline A & 01 & 03,4 \\
\hline B1 & 03 & 10,3 \\
\hline B2 & 04 & 13,8 \\
\hline $\mathrm{C} 1$ & 11 & 37,9 \\
\hline $\mathrm{C} 2$ & 08 & 27,6 \\
\hline D-E & 02 & 06,9 \\
\hline
\end{tabular}

Com relação ao tempo de concessão da cadeira de rodas, $37,9 \%$ dos participantes receberam o equipamento há mais de dois anos, apenas um declarou ter recebido há menos que seis meses. E, quanto ao período de uso da cadeira de rodas durante um dia, constatou-se que 34,5\% permaneciam de 3 a 6 horas na cadeira, 6,9\% utilizavam a cadeira menos que uma hora por dia, e $13,8 \%$ ficavam 
Cavalcanti A, et al. Percepção dos responsáveis de crianças e adolescentes. Rev Ter Ocup Univ São Paulo. 2018 jan/abr:,29(1):27-33.

posicionados no equipamento mais de 10 horas em um período de 24 horas (Tabela 2).

Tabela 2 - Tempo de concessão e tempo de uso em um dia $(n=29)$

\begin{tabular}{lcc}
\hline Variável & $\mathbf{n}$ & $\mathbf{\%}$ \\
\hline Tempo de uso durante um dia & & \\
Menos de 1 hora & 02 & 06,9 \\
1-3 horas & 07 & 24,1 \\
3-6 horas & 10 & 34,5 \\
6-10 horas & 06 & 20,7 \\
Acima de 10 horas & 04 & 13,8 \\
\hline
\end{tabular}

\section{Tempo de Concessão}

Menos que 6 meses

6-12 meses

12-18 meses

$01 \quad 03,4$

18-24 meses

$10 \quad 34,5$

Acima de 24 meses

$06 \quad 20,7$

$11 \quad 37,9$

O motivo da solicitação de cadeira de rodas apontado pelos pais/responsáveis das crianças e adolescentes foi identificado como tendo uma única causa para 51,7\% dos participantes e como tendo mais de um motivo para os demais 48,3\%. Quando identificado apenas um motivo para a solicitação da prescrição, 46,7\% apontaram a mobilidade (Tabela 3).

Tabela 3 - Motivo da prescrição da cadeira de rodas $(n=29)$

\begin{tabular}{lcc}
\hline Variável & n & $\mathbf{\%}$ \\
\hline Mobilidade & 21 & 72,4 \\
Gerenciamento da postura & 19 & 65,5 \\
Transporte & 13 & 44,8 \\
Facilidade para uso & 10 & 34,5 \\
\hline
\end{tabular}

Já as questões discursivas sobre o conforto ou desconforto percebido em algum momento ao longo de um dia em que o equipamento está sendo usado pela criança ou adolescente, $86,2 \%$ dos pais/responsáveis apontaram inferir desconforto de seus filhos em diferentes situações agrupadas nas categorias: 'uso por mais de duas horas' (48,3\%), 'acessório ou tamanho inadequado' à necessidade de seus filhos (27,6\%), 'perda do controle motor de tronco' $(13,8 \%)$, 'mobilidade na rua' $(13,8 \%)$ e 'atividades' $(10,3 \%)$ (Tabela 4). O conforto foi apontado por $48,3 \%$ dos pais/ responsáveis nas categorias: 'mobilidade' (31\%), 'lazer' (31\%), 'educação' $(13,8 \%)$, 'alimentação' (10,3\%), sono/ descanso $(6,9 \%)$ e em outra categoria com menor percentual como demonstrado na Tabela 4.

Tabela 4 - Apontamentos dos pais/responsáveis sobre Conforto-Desconforto e Satisfação-Insatisfação em relação ao equipamento de seus filhos quando em uso $(n=29)$

\begin{tabular}{lcc|lcc}
\hline Variável Conforto & $\mathbf{n}$ & $\mathbf{\%}$ & Variável Desconforto & $\mathbf{n}$ & $\mathbf{\%}$ \\
\hline Mobilidade & 09 & 31,0 & Uso por mais de $2 \mathrm{~h}$ & 48,3 \\
Lazer & 09 & 31,0 & Acessórios ou tamanho inadequado & 08 & 27,6 \\
Educação & 04 & 13,8 & Perda do controle de tronco & 04 & 13,8 \\
Alimentação & 03 & 10,3 & Mobilidade na rua & 04 & 13,8 \\
Sono/Descanso & 02 & 06,9 & Atividade & 03 \\
Mesa de atividade & 01 & 03,4 & & $\mathbf{0}, 3$ \\
\hline Variável Satisfação & $\mathbf{n}$ & $\mathbf{\%}$ & Variável Insatisfação & $\mathbf{n}$ \\
\hline Fornece mobilidade & 20 & 69,0 & Acessórios ruins & 21 \\
Melhora a postura & 15 & 51,7 & Tamanho inadequado & 12 \\
Acessórios & 10 & 34,5 & Freio ruim & 41,4 \\
Tamanho adequado & 04 & 13,8 & Pneu esvazia & 37,9 \\
Fácil limpeza & 03 & 10,3 & Difícil montar/desmontar & 11 \\
Apóia a função & 02 & 06,9 & Pesada & 34,5 \\
Peso adequado & 02 & 06,9 & Sem queixa & 10 \\
Material resistente & 01 & 03,4 & & 34,5 \\
Segura & 01 & 03,4 & & 13,8 \\
Nada a declarar & 01 & 03,4 & & 10,3 \\
\hline
\end{tabular}


E, sobre os itens que os pais/responsáveis apontam como satisfeitos e insatisfeitos em relação à cadeira de rodas, as categorias para satisfação foram: 'fornece mobilidade' (69\%), 'melhora a postura' $(51,7 \%)$, 'acessórios' (34,5\%), 'tamanho adequado' (13,8\%), 'fácil limpeza' (10,3\%), 'apoia a função' $(6,9 \%)$ e 'peso adequado' $(6,9 \%)$. Outras categorias foram apontadas apenas por um pai/responsável como pode ser verificado na Tabela 4. Para os itens descritos como elementos de insatisfação da criança/adolescente na cadeira de rodas, as categorias identificadas foram: 'acessórios ruins' (cinto de segurança, cinto torácico ou itens da cadeira como apoio de cabeça e apoio de pés) $(72,4 \%)$, 'tamanho inadequado' (41,4\%), 'freio ruim' (37,9\%), 'pneu esvazia' (34,5\%), 'difícil montar/desmontar' (34,5\%) e 'pesada' $(13,8 \%)$. Três participantes declararam não terem itens de insatisfação (Tabela 4).

\section{DISCUSSÃO}

As cadeiras de rodas são equipamentos de tecnologia assistiva rotineiramente usados para o fornecimento de mobilidade, para a adequação da postura sentada e para o conforto de crianças que não deambulam e apresentam a espasticidade como um dos sinais clínicos, como nos casos de paralisia cerebral e de lesões não traumáticas ${ }^{11}$. Dolan e Henderson ${ }^{1}$ descrevem que usuários típicos de cadeira de rodas são jovens com paralisia cerebral e pessoas com desordens progressivas. Corroborando com esses apontamentos, neste estudo a maioria $(82,8 \%)$ das crianças e adolescentes usuários de cadeira de rodas possuem diagnóstico clínico de paralisia cerebral.

Ao receber uma cadeira de rodas de um serviço de concessão, principalmente aqueles ofertados por políticas públicas, a Organização Mundial de Saúde (OMS) preconiza que acompanhamentos e revisões periódicas, por parte da equipe que prescreveu e entregou a cadeira de rodas junto aos usuários, são necessários para verificar a continuidade de sua adequação, reforçar o treinamento ministrado no momento do recebimento do equipamento e fornecer suporte ${ }^{12}$. Esta estratégia de prestação de serviço é nomeada pela OMS como 'visitas de acompanhamento' e é caracterizada como uma oportunidade para verificar se o equipamento está funcionando adequadamente e se está apropriada às necessidades do usuário. Quando a equipe identifica um descompasso entre as demandas do usuário e a cadeira de rodas fornecida, uma nova prescrição deve ser realizada ${ }^{12}$.

Para crianças e adolescentes, as 'visitas de acompanhamento' são valiosas em virtude das mudanças diárias de suas necessidades, associadas ao seu crescimento e ao seu desenvolvimento ${ }^{13}$. A literatura sugere que para crianças seja realizada uma nova avaliação a cada seis meses, devendo ser iniciada a 'visita de acompanhamento' após as seis primeiras semanas da data de entrega da cadeira.

Neste estudo, 93,1\% das crianças e adolescentes possuem cadeira de rodas há mais de doze meses, incluindo aqueles que relataram já usar o equipamento há mais de dois anos. Essa observação reforça os achados a respeito dos itens que geram insatisfação dos pais/responsáveis em relação à cadeira: comprometimento (desgaste) de acessórios ou parte deles como o cinto de segurança, o cinto torácico, ou itens da cadeira como o apoio de cabeça e apoio de pé; tamanho da cadeira inadequado para seu filho(a); sistema de freio que não funciona (trava quebrada ou que não desliza), pneu se esvazia regularmente (sendo necessária a calibragem dos pneus por 2-3 vezes na semana), e a dificuldade de manusear a estrutura para abrir (montar) e fechar (desmontar para guardar) devido ao desgaste das peças ou falta de lubrificação.

Neste sentido, 'visitas de acompanhamento' garantiriam a qualidade do serviço ofertado. Dolan e Henderson ${ }^{1}$ concluem em um estudo realizado sobre adequação da postura sentada em cadeira de rodas que o desgaste natural do equipamento e a evolução no quadro clínico do usuário levam a substituição do sistema de assento (almofada) da cadeira de rodas a cada 3 a 5 anos em média e da estrutura da cadeira a cada 2 anos e 5 meses. Nas orientações da British Society of Rehabilitation Medicine, uma equipe multidisciplinar deve avaliar a cadeira de rodas cada 2 a 3 anos $^{14}$.

Quando se pontuando sobre o tempo de uso da cadeira em um dia, é consenso que quanto maior o tempo, maior são as considerações a respeito do risco de desconforto, fadiga e aparecimento de lesões por pressão ${ }^{13}$. Um período de tempo prolongado sobre uma cadeira de rodas deve estar associado a sistemas de assento especializados que visam ao alívio da pressão e garantem o conforto. Neste estudo, $65,5 \%$ dos pais/responsáveis relataram que suas crianças ou adolescentes usam o equipamento por três horas no mínimo. E, neste sentido, deve ser investigado o sistema de adequação postural dos participantes, pois apoio adicional para a adequação pode melhorar o conforto, aumentar a resistência à fadiga para sentar e garantir um sentar ereto para atividades de alimentação, especialmente se o usuário tiver paralisia cerebral $^{13}$.

Além da adequação postural que conduz ao conforto e à funcionalidade, uma cadeira de rodas fornece mobilidade. Rodby-Bousquet e Hagglund ${ }^{15}$ 
destacam que quase um terço das crianças com paralisia cerebral é dependente de equipamentos para a locomoção. Cury, Figueiredo e Mancini ${ }^{16}$ explicitam que crianças e adolescentes com paralisia cerebral rotineiramente precisam de equipamento de assistência e treino para mobilidade funcional, definido de acordo com as habilidades do usuário e com o ambiente em que a mobilidade irá ser desempenhada. Uma cadeira de rodas pode prover deslocamento, permitindo envolvimento em tarefas desejadas e/ou necessárias ao longo de um dia, ampliando a oportunidade de envolvimento em atividades e na participação. Para os participantes desse estudo o principal motivo da solicitação da cadeira de rodas envolve mobilidade.

Para mobilidade é necessário o gerenciamento da postura. E, quando o usuário se mantém em simetria, equilíbrio e estabilidade corporal, o conforto é obtido ${ }^{1}$. O conforto do usuário confirma as escolhas feitas pela equipe de prescrição a respeito da cadeira de rodas e sua eficácia para uma adequação postural ${ }^{17}$ com escolhas baseadas na avaliação e na escuta das necessidades e desejos dos usuários. Os pais/responsáveis apontaram as atividades de mobilidade (fora de casa), lazer (assistir televisão e brincar), alimentação, educação (uso na escola) e apontaram o emprego de mesa de atividade (mesa bandeja) como as situações em que percebiam conforto de seus filhos na cadeira de rodas. Os relatos são equivalentes às funções esperadas para o dispositivo. Engström ${ }^{18}$ pontua que uma cadeira de rodas fornece maior conforto se uma mesa de atividades for posicionada para apoio dos membros superiores e assistência do controle de tronco.

As situações de desconforto envolveram relatos dos participantes sobre as crianças e adolescentes permanecerem sentados em um período superior a duas horas, ou quando perdem o controle postural ao se movimentarem no equipamento, na mobilidade fora de casa e nos casos de inadequação de acessórios às necessidades ou sua inexistência no dispositivo. A permanência de crianças e adolescentes com desordens motoras graves em cadeira de rodas por longos períodos é apontada como pertinente somente se o dispositivo tem suportes adicionais para as demandas que cada usuário necessita para manterse em equilíbrio, estável e com conforto ${ }^{12}$.

É importante destacar ainda que uma cadeira demandará alterações ao longo do tempo em que estará em uso, acompanhando o desenvolvimento de seu usuário e as mudanças de suas habilidades e necessidades ${ }^{1}$. O desconforto pode levar a movimentação anormal, à perda da simetria corporal, à alteração de tônus, à diminuição do envolvimento em atividades e ao abandono do equipamento ${ }^{17}$.

Os acessórios são componentes que auxiliam na manutenção da estabilidade do usuário na cadeira de rodas $^{11}$. Funções especializadas como tilt (inclinação do conjunto assento/encosto sobre o eixo da cadeira de rodas), modelos diversos de cintos torácicos e pélvicos, possibilidade de regulagem em altura e profundidade de apoio de pés, apoio de braços e apoio de cabeça são opções disponíveis em diferentes modelos de cadeiras de rodas, por exemplo. Somando-se as opções de acessórios para auxiliar na manutenção do conforto, ainda se têm as especificidades dos componentes e dos sistemas de assentos. No estudo conduzido por Dolan e Henderson ${ }^{1}$ a respeito do perfil de pacientes e as especificações de cadeira de rodas, os autores registraram que em torno de $25 \%$ estavam usando cadeira de rodas manual com tilt e/ ou recline (aumento do ângulo entre assento e encosto), destacando a importância destes.

\section{CONSIDERAÇÕES FINAIS}

Este estudo mostra a percepção dos pais/ responsáveis sobre o motivo da solicitação de cadeira de rodas para seus filhos e descreve como percebem o conforto e o desconforto quando suas crianças/adolescentes usam a cadeira e quais itens apontam satisfação ou não no equipamento concedido. Os achados demonstram que os participantes identificam que a cadeira de rodas é designada para mobilidade. Com a observação de apontamentos de um grupo significativo para gerenciamento da postura, facilidade para uso e transporte, identificações que se tornam distintas quando são relacionadas às características funcionais das crianças/adolescentes.

A maioria dos pais/responsáveis relatou perceber desconforto de sua criança/adolescente na cadeira de rodas e apontou insatisfação de itens no equipamento relacionados aos seus acessórios. Diante destes dados, verifica-se que é importante rever o processo de concessão da cadeira de rodas e o acompanhamento do usuário de forma mais próxima, buscando dar qualidade e oportunidade de melhor uso do equipamento concedido.

Frente às observações de desconforto e insatisfação, novos estudos são sugeridos junto às crianças/adolescentes para investigação sobre a possibilidade de abandono do equipamento, sobre a capacidade motora que possuem, sobre sua relação com o tipo do sistema de adequação postural da cadeira de rodas que possuem e sobre a necessidade ou não de adequação adicional tanto do assento quanto de outro item do equipamento. 
Contribuição dos autores: Alessandra Cavalcanti: concepção do estudo, coleta e análise de dados, redação e revisão do texto. Eduarda Caroline Marques Borges: coleta e análise de dados, redação do texto. Bruna Correa Magalhães: coleta e análise de dados. Edinara Kososki: coleta e análise de dados, redação do texto. Alberto Luiz Aramaki: coleta e análise de dados, redação do texto. Simone Gonçalves Silva e Silva: análise de dados.

\section{REFERÊNCIAS}

1. Dolan MJ, Henderson GI. Patient and equipment profile for wheelchair seating clinic provision. Disabil Rehabil Assist Technol. 2014;9(2):136-43. doi: 10.3109/17483107.2013.807441.

2. Cooper RA. Guest editorial: wheelchair research progress, perspectives, and transformation. J Rehabil Res Dev. 2012;49(1):1-6. http://dx.doi.org/10.1682/ JRRD.2011.10.0199.

3. Mcclure LA, Boninger ML, Oyster ML, Williams S, Houlihan B, Lieberman JA, Cooper RA. Wheelchair repairs, breakdown, and adverse consequences for people with traumatic spinal cord injury. Arch Phys Med Rehabil. 2009;90:2034-8. doi: 10.1016/j.apmr.2009.07.020.

4. Toro ML, Worobey L, Boninger ML, Cooper RA, Pearlman J. Type and frequency of reported wheelchair repairs and related adverse consequences among people with spinal cord injury. Arch Phys Med Rehabil. 2016;97(10):1753-60. doi: 10.1016/j.apmr.2016.03.032.

5. Askari S, Kirby L, Parker K, Thompson MK, O’Neill J. Wheelchair propulsion test: development and measurement properties of a new test for manual wheelchair users. Arch Phys Med Rehabil. 2013;94:1690-8. doi: 10.1016/j. apmr.2013.03.002.

6. McDonald R, Surtees R, Wirz S. A comparison between parents' and therapists' views of their child's individual seating systems. Int J Rehabil Res. 2003;26(3):235-43. doi: 10.1097/01.mrr.0000088449.78481.7c.

7. Karp G. Chossing a wheelchair. A guide for optimal independence. USA: O’Reilly; 1998.

8. Magalhaes BC, Cavalcanti A. Mapeamento de cadeira de rodas no município de Uberaba/MG. Relatório final de projeto de pesquisa BIC/FAPEMIG. Uberaba: UFTM; 15 jul. 2015.
9. Associação Brasileira das Empresas de Pesquisa. Critério de classificação econômica Brasil [citado 11 ago. 2014]. Disponível em: http://www. abep.org/novo/.

10. Bardin LL. Análise de conteúdo. 3a ed. Rio de Janeiro: Edições70; 2010.

11. Cimolin V, Avellis M, Piccinini L, Corbetta C, Cazzaniga A, Turconi AC, Galli M. Comparison of two pelvic positioning belt configurations in a pediatric wheelchair, assistive technology. Assist Technol. 2013;254:240-6. doi: 10.1080/10400435.2013.778916.

12. Frost S, Mines K, Noon J, Scheffler E, Stoeckle RJ. Wheelchair service training package: basic level. Malta: World Health Organization; 2012.

13. Khasnabis $\mathrm{C}$, Mines $\mathrm{K}$. Wheelchair service training package: intermediate level. Malta: World Health Organization; 2013.

14. British Society of Rehabilitation Medicine. Specialized wheelchair seating national clinical guidelines. London; 2004 [cited 2016 July 01]. Available from: http://www.bsrm.org.uk/ publications/publications.

15. Rodby-Bousquet E, Hagglund G. Use of manual and powered wheelchair in children with cerebral palsy: a cross-sectional study. BMC Pediatrics. 2010;10:59. doi: 10.1186/1471-2431-10-59.

16. Cury VCR, Figueiredo PRP, Mancini MC. Environmental settings and families' socioeconomic status influence mobility and the use of mobility devices by children with cerebral palsy. Arq Neuropsiquiatr. 2013;71(2):100-5. http://dx.doi. org/10.1590/S0004-282X2013005000003.

17. Cavalcanti A, Galvão C, Campos MAAD. Cadeira de rodas e sistema de adequação postural. In: Cavalcanti A, Galvão C. Terapia ocupacional: fundamentação \& prática. Rio de Janeiro: Guanabara Koogan; 2007. p.451-61.

18. Engström B. Ergonomic seating a true challenge. Wheelchair seating \& mobility principles. Stallarholmen: Posturalis Books; 2002. p.91-120.

Recebido em: 20.11 .16

Aceito em: 21.05 .18 